\title{
Leishmania (Leishmania) chagasi- infected mice as a model for the study of glomerular lesions in visceral leishmaniasis
}

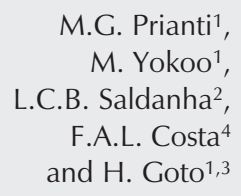

M.G. Prianti ${ }^{1}$,

M. Yokoo ${ }^{1}$,

L.C.B. Saldanha

F.A.L. Costa ${ }^{4}$

and $\mathrm{H}$. Goto ${ }^{1,3}$

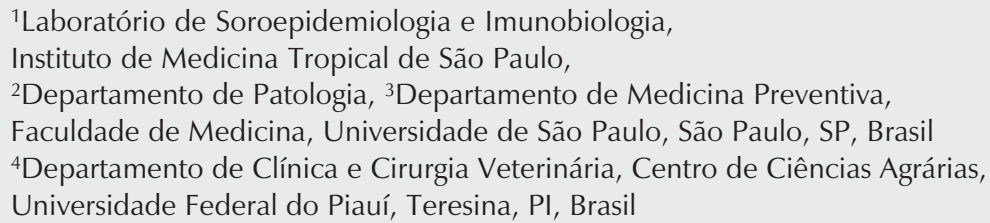

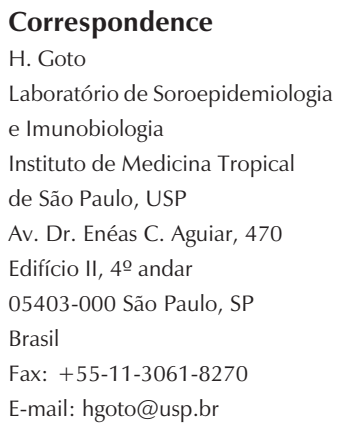

Publication supported by FAPESP. $\ldots \ldots \ldots \ldots \ldots$

Received January 10, 2007 Accepted April 3, 2007

\begin{abstract}
Renal involvement in visceral leishmaniasis (VL) is very frequent but the pathogenesis of this nephropathy is poorly understood. In previous studies using dogs with VL we have detected new immunopathological elements in the glomeruli such as T cells and adhesion molecules. Although Leishmania (Leishmania) chagasi-infected dogs and hamsters are considered to be good models for VL, their use is limited for immunopathologic studies. The use of isogenic mouse strains susceptible to $L$. $(L$.) chagasi infection was an alternative but, on the other hand, the renal lesions of these animals have not yet been characterized. Thus, our purpose in the present study was to characterize mice infected with $L$. $(L$.) chagasi as a suitable model to study VL nephropathy. Kidney samples were obtained from control mice $(\mathrm{N}=12)$ and from $\mathrm{BALB} / \mathrm{c}$ mice $(\mathrm{N}=24)$ injected intraperitoneally with 20 million L. (L.) chagasi amastigotes 7, 15, and 30 days after injection and processed for histopathological studies and detection of IgG deposits. Glomerular hypercellularity was clearly visible and, upon Mason's trichrome and periodic acid methenamine silver staining, a pattern suggestive of mesangial proliferative glomerulonephritis was observed in mice with VL. Time-dependent IgG deposits were also seen in infected mice. We consider $L$. $(L$. ) chagasi-infected mice to be a suitable model for studies of the immunopathogenesis of glomerular lesions in VL.
\end{abstract}

Key words

- Visceral leishmaniasis

- Glomerulonephritis

- Mice

- Model

- Leishmania (Leishmania)

chagasi
Visceral leishmaniasis (VL) is caused by the protozoa Leishmania (Leishmania) chagasi in Brazil, it is fatal if not treated, and is characterized by fever, hepatosplenomegaly, anemia, hypergammaglobulinemia, and leukopenia. Several organs in addition to those rich in mononuclear phagocytes are affected in VL (1). Renal involvement is very frequent and the glomerular lesions are related to the severity of the disease (2). In the glomerulonephritis (GN) occurring in VL, glomerular alterations have been considered to be a consequence of the immune response and immune complex deposition 
has been thought to be the mechanism of injury (3-6). However, in our previous studies on dogs with VL, we have detected new imunopathological elements in renal changes in VL, such as $\mathrm{T}$ cells and adhesion molecules $(7,8)$. In addition, different cytokines have also been shown to be involved in the pathogenesis of nephropathy in malaria, another protozoan disease (9). Therefore, further studies on the pathogenic mechanisms involved in the GN of VL are needed. Since canine and human VL share similar disease manifestations, dogs represent an appropriate animal model for the human disease (10). In addition, golden hamsters (Mesocricetus auratus) infected with $L$. donovani or $L$. chagasi are also considered to be an excellent VL model (11). However, their use is limited for immunopathological studies, mainly due to the lack of available antibodies for immunological markers (10). An alternative is to use inbred mouse strains susceptible to $L$. (L.) chagasi infection.

A susceptible model of VL is $L$. donova$n i$-infected BALB/c mice in which the infection progresses during the first two weeks, when it becomes controlled by the host immune response (12). There are studies on the immunity of this model (13), but nephropathy has not been studied. Therefore, we experimentally infected BALB/c mice using $L$. (L.) chagasi amastigotes to characterize a model suitable for the study of VL nephropathy.

Male BALB/c mice supplied by the Animal Breeding Facility of the Medical School of the University of São Paulo were maintained in the Animal Facility of the Tropical Medicine Institute of São Paulo, University of São Paulo. Mice were injected intraperitoneally with either $2 \times 10^{7}$ purified $L$. (L.) chagasi (MHOM/BR/72/strain 46) amastigotes according to Dwyer (14) or RPMI 1640 medium (Gibco BRL, Gaithersburg, MD, USA) as control, and sacrificed at 7, 15, and 30 days post-infection (PI). At the time of sacrifice, a kidney was removed and fixed in formalin, embedded in paraffin, and 5- $\mu \mathrm{m}$ thick sections were prepared. To quantitatively evaluate the glomerular cell number, renal tissue sections were obtained from 24 infected and 12 control-BALB/c mice and stained with hematoxylin-eosin.

During the course of the experiment, glomerular hypercellularity was observed from 7 through 30 days PI in infected animals (Figure 1A) compared with non-infected ones (Figure 1B). Interstitial alterations were not observed in either infected or non-infected animals (data not shown). To further study the histopathological pattern of glomerular changes, sections from samples fixed in Bouin and embedded in paraffin were stained with Masson's trichrome and periodic acid methenamine silver (PAMS). In this analysis, most of the glomeruli from infected animals showed an increased number of glomerular cells, occupying almost the total glomerular area. However, the wall of the glomerular capillaries was normal (Figure 1C). These findings suggest a mesangial proliferative pattern of GN in this model. The control group presented normal cellularity as well as normal glomerular capillary walls (Figure 1D). Probably due to the use of an inbred mouse strain, the histopathological pattern was homogeneous in all samples and the alterations were present in all. Morphometric analysis was performed to confirm the hypercellularity of the glomeruli. The number of cells was counted in 20 glomeruli in each section stained with hematoxylineosin to obtain the number of cells/glomerulus. A significantly larger number of glomerular cells was observed in the infected animals when compared with the non-infected control animals at 7,15 and 30 days $\mathrm{PI}(\mathrm{P}<0.05$; Figure 1E).

In this model, hypercellularity seems to be independent of the inoculation route, since when $\mathrm{BALB} / \mathrm{c}$ mice were infected by the intravenous route the results were similar (data not shown). The GN showing a mesangial proliferative pattern in this model re- 
flects one of the patterns seen in human, canine and hamster VL $(3,4,15)$. The histopathological changes in glomerular lesions systematically described in canine VL have shown different patterns of GN but with a predominance of proliferative patterns including mesangial proliferative pattern (15). It is also known that one of the features of human GN is the proliferation of mesangial cells and consequent glomerular hypercellularity $(4,16)$. The pathogenic process leading to hypercellularity found in the glomeruli in VL is one of the targets for further studies upon the establishment of a good experimental model, which was the objective of the present study.

Since in studies on VL nephropathy immunoglobulins have always been detected in renal lesions (3-6) this aspect was also considered in our samples. We analyzed the presence of $\mathrm{IgG}$ deposit on cryosections of the kidney by indirect immunofluorescence using a biotin-conjugated rabbit anti-mouse IgG antibody and streptavidin-fluorescein isothiocyanate system (Dako Corporation, Carpinteria, CA, USA). The complement fraction $\mathrm{C} 3 \mathrm{~b}$ deposit on frozen sections was also evaluated by the same protocol using a polyclonal rabbit anti-mouse $\mathrm{C} 3 \mathrm{~b}$ antibody produced in our laboratory (17). The sections were then examined under a fluorescent microscope (Carl Zeiss, Göttingen, Germany). The intensity of the deposits was evaluated semi-quantitatively and is reported as scores from 0 (negative) to 4 (intense staining throughout all glomeruli) (16) upon analysis of at least 20 glomeruli per sample.

When searching for IgG deposits, we observed the time-dependence of its presence in glomeruli. Although discrete, a baseline deposit was observed in most of the infected (Figure 2B) and non-infected animals (Figure 2C) throughout the experimental period, but the significant fluorescent deposit (Figure 2A) was only observed at 7 days PI when it was significantly greater than in non-infected controls. This observa- tion could be confirmed when semi-quantitative analysis of the intensity of the deposits of IgG in the glomeruli was performed. This revealed a significant increase only at 7 days of infection in relation to the control and to animals examined at the other post-infection times $(\mathrm{P}<0.05$; Figure 2D). Deposits of the $\mathrm{C} 3 \mathrm{~b}$ fraction of complement was analyzed in glomeruli in 12 non-infected and 24 infected

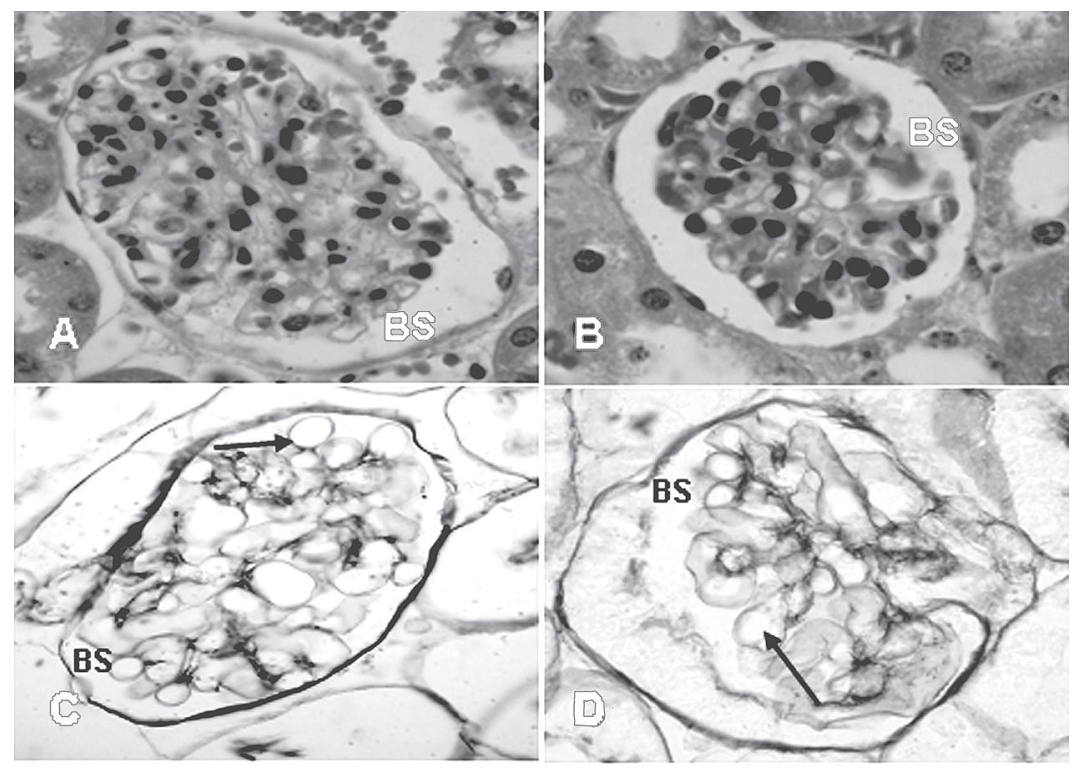

E

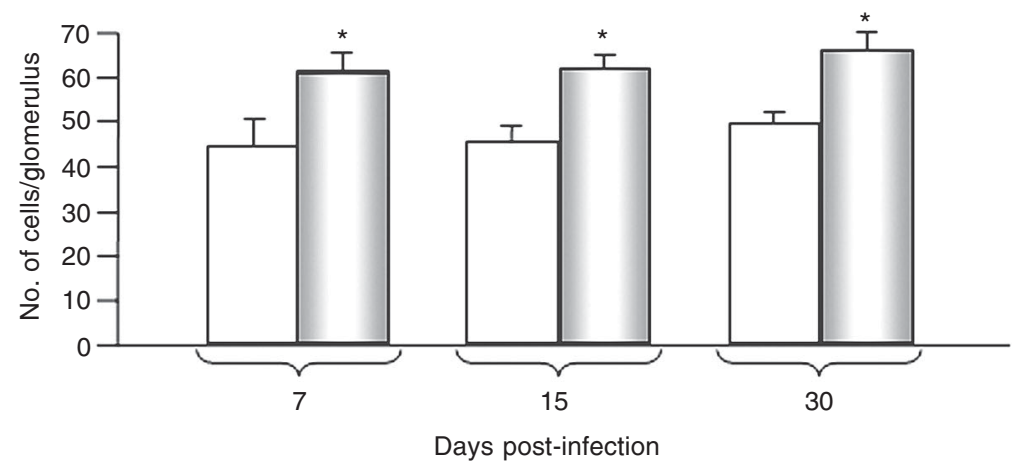

Figure 1. Kidney sections stained with hematoxylin-eosin. $A$, Glomerulonephritis in BALB/c mice infected with Leishmania $(L$.) chagasi. Hypercellularity in infected mice with glomerular tuft occupying almost the whole Bowman space (BS) (40X). B, Normal cells in glomeruli from control non-infected mice (20X). Kidney stained with periodic acid methenamine silver (40X). C, Glomerulonephritis in BALB/c mice infected with $L$. (L.) chagasi. Hypercellularity in glomerulonephritis and normal glomerular capillary wall (arrow) in infected mice. $D$, Normal cellularity in glomeruli of a control non-infected mouse. E, Cell number per glomerulus (mean $\pm \mathrm{SD}$ ) in BALB/c mice non-infected $(\mathrm{N}=4)$ and infected with $L$. (L.) chagasi $(\mathrm{N}=8)$ at 7,15 , and 30 days post-infection. Non-infected = open columns; infected $=$ filled columns. ${ }^{*} P<0.05$ compared to non-infected control (ANOVA on ranks and StudentNewman-Keuls test). 
animals. Subtle granular fluorescence was seen in glomeruli in only 5 of 24 infected animals at 7 and 30 days PI and was totally absent in control animals. The very subtle deposit of $\mathrm{C} 3 \mathrm{~b}$ and in a restricted number of infected animals suggests that complement does not have an important participation in the pathogenesis of GN caused by infection with $L$. (L.) chagasi. Furthermore, similar findings were observed in hamsters with VL in our previous studies (18).

The present findings support the use of a mouse model for the study of VL glomeru-


Figure 2. IgG deposits (arrow) on the glomerular capillary wall of an infected mouse. A, Presence of fluorescence of moderate intensity in the glomerular capillary wall of infected animals at 7 days post-infection (PI). B, At 15 days $\mathrm{PI}$, presence of discrete fluorescence. $C$, Absence of IgG deposits in glomeruli of a control non-infected mouse. Indirect immunofluorescence (20X). $D$, Semi-quantitative data (score) of IgG deposits in glomeruli from BALB/c mice non-infected $(\mathrm{N}=4)$ and infected with Leishmania $($ L. $)$ chagasi $(\mathrm{N}=8)$ at 7,15 , and 30 days $\mathrm{PI}$. Non-infected $=$ open columns; infected $=$ filled columns. ${ }^{*} \mathrm{P}<0.05$ compared to non-infected control (ANOVA on ranks and Student-Newman-Keuls test). lopathy since similar observations were made before in hamsters with VL (18) which has been considered to be a good animal model of VL. The fact that IgG apparently participates only in the early phase of infection, the almost absent evidence of participation of complement in the pathologic process but the persistence of hypercellularity in subsequent phases as shown here point to the necessity of further studies of the pathogenic mechanisms of these lesions. Such studies may imply different approaches focusing on different cell populations such as mesangial cells, lymphocytes, different molecules involved in inflammatory and immunological processes, and different pathogenic processes involving immunoglobulins. For these approaches the availability of reagents and some gene-targeted animal strains, and isogenic animals to allow cell transfer experiments are fundamental, and these requirements may be satisfied by using the mouse model.

To the best of our knowledge, this is the first investigation using $\mathrm{BALB} / \mathrm{c}$ mice injected intraperitoneally with $L$. (L.) chagasi to study GN, and our data indicate that it is a good model for the study of glomerular alterations mainly of a proliferative pattern in VL. It will be a useful model mainly for the study of immunopathogenic mechanisms considering the availability of reagents and gene-targeted mouse strains for immunological studies.

\section{Acknowledgments}

We thank the Histopathology Laboratory of the Department of Pathology, Faculdade de Medicina, Universidade de São Paulo, for technical assistance.

\section{References}

1. Duarte MIS. Patologia das principais doenças tropicais no Brasil. Leishmaniose visceral (Calazar). In: Brasileiro Filho G (Editor), Bogliolo Patologia. 6th ed. Rio de Janeiro: Editora Guanabara
Koogan; 2000. p 1215-1227.

2. Duarte MI, Silva MR, Goto H, Nicodemo EL, Amato Neto V. Interstitial nephritis in human kala-azar. Trans R Soc Trop Med Hyg 1983; 
77: 531-537.

3. Sartori A, De Oliveira AV, Roque-Barreira MC, Rossi MA, CamposNeto A. Immune complex glomerulonephritis in experimental kalaazar. Parasite Immunol 1987; 9: 93-103.

4. de Brito T, Hoshino-Shimizu S, Neto VA, Duarte IS, Penna DO. Glomerular involvement in human kala-azar. A light, immunofluorescent, and electron microscopic study based on kidney biopsies. Am J Trop Med Hyg 1975; 24: 9-18.

5. Poli A, Abramo F, Mancianti F, Nigro M, Pieri S, Bionda A. Renal involvement in canine leishmaniasis. A light-microscopic, immunohistochemical and electron-microscopic study. Nephron 1991; 57: 444-452.

6. Weisinger JR, Pinto A, Velazquez GA, Bronstein I, Dessene JJ, Duque JF, et al. Clinical and histological kidney involvement in human kala-azar. Am J Trop Med Hyg 1978; 27: 357-359.

7. Costa FA, Guerra JL, Silva SM, Klein RP, Mendonca IL, Goto H. CD4(+) T cells participate in the nephropathy of canine visceral leishmaniasis. Braz J Med Biol Res 2000; 33: 1455-1458.

8. Costa FAL. Patologia e imunopatogenia da nefropatia da leishmaniose visceral canina [Thesis]. São Paulo: Faculdade de Medicina Veterinária e Zootecnia, USP; 2001

9. Sinniah R, Rui-Mei L, Kara A. Up-regulation of cytokines in glomeruIonephritis associated with murine malaria infection. Int J Exp Pathol 1999; 80: 87-95.

10. Carrera L, Fermin ML, Tesouro M, Garcia P, Rollan E, Gonzalez JL, et al. Antibody response in dogs experimentally infected with Leishmania infantum: infection course antigen markers. Exp Parasitol
1996; 82: 139-146.

11. Requena JM, Soto M, Doria MD, Alonso C. Immune and clinical parameters associated with Leishmania infantum infection in the golden hamster model. Vet Immunol Immunopathol 2000; 76: 269 281.

12. Murray HW, Stern JJ, Welte K, Rubin BY, Carriero SM, Nathan CF. Experimental visceral leishmaniasis: production of interleukin 2 and interferon-gamma, tissue immune reaction, and response to treatment with interleukin 2 and interferon-gamma. J Immunol 1987; 138 : 2290-2297.

13. Goto $\mathrm{H}$, Lindoso JA. Immunity and immunosuppression in experimental visceral leishmaniasis. Braz J Med Biol Res 2004; 37: 615623.

14. Dwyer DM. Antibody-induced modulation of Leishmania donovani surface membrane antigens. J Immunol 1976; 117: 2081-2091.

15. Costa FA, Goto H, Saldanha LC, Silva SM, Sinhorini IL, Silva TC, et al. Histopathologic patterns of nephropathy in naturally acquired canine visceral leishmaniasis. Vet Pathol 2003; 40: 677-684.

16. Tisher CC, Brenner BM. Renal pathology with clinical and functional correlations. 2nd edn. Philadelphia: JB Lippincott Company; 1994.

17. Laurenti MD, Corbett CE, Sotto MN, Sinhorini IL, Goto H. The role of complement in the acute inflammatory process in the skin and in host-parasite interaction in hamsters inoculated with Leishmania (Leishmania) chagasi. Int J Exp Pathol 1996; 77: 15-24.

18. Mathias R, Costa FA, Goto H. Detection of immunoglobulin $\mathrm{G}$ in the lung and liver of hamsters with visceral leishmaniasis. Braz J Med Biol Res 2001; 34: 539-543. 